www.jmscr.igmpublication.org

Impact Factor (SJIF): 6.379

Index Copernicus Value: 71.58

ISSN (e)-2347-176x ISSN (p) 2455-0450

crossrefDOI: https://dx.doi.org/10.18535/jmscr/v6i7.185

Journal Of Medical Science And Clinical Research

\title{
POEMS Syndrome: An Atypical Presentation
}

Authors

\section{Dr Judah Nijas Arul ${ }^{1}$, Dr V. Santosh Kumar², Dr S.R. Ramakrishnan ${ }^{3}$, Dr Mohini Singh ${ }^{4}$, Dr Vaasanthi $\mathbf{R}^{\mathbf{5}}$, Dr George Eapen ${ }^{6}$}

${ }^{1}$ Junior Resident, ${ }^{2,6}$ Senior Resident, ${ }^{3}$ Professor, ${ }^{4}$ Associate Professor, ${ }^{5}$ Assistant Professor Department of General Medicine, Sri Ramachandra Medical College \& Research Institute, Chennai

Tamil Nadu, India

Corresponding Author

\section{Dr S.R. Ramakrishnan}

Address: 2/79, MGR Street, Srinivasapuram, Iyappanthangal, Chennai, Tamil Nadu, India 600056 Email:drsrk_71@yahoo.com, Phone: 9444912452

\begin{abstract}
POEMS Syndrome is a rare disorder with multiple entities constituting it and it commonly presents in the fifth to sixth decade of life with a male predominance. We report a case of a 79-year-old male from South India who initially presented with vague complaints of tiredness and weakness for the past few months along with thrombocytopenia. Further investigations led to the discovery of the presence of Monoclonal plasma cell disorder with M Band in the Beta 1 region, severe sensorimotor polyneuropathy and other findings which fulfilled the criteria for POEMS syndrome.

Keywords: POEMS Syndrome, Multiple Myeloma, Vitamin B12 deficiency.
\end{abstract}

\section{Introduction}

POEMS is a rare entity and requires a high index of suspicion by the treating physician as it manifests with diverse clinical features. It is thought to be the paraneoplastic presentation of the underlying plasma cell disorder.

We present the following case as the clinical presentation of the patient was rather vague, his complaints would have been easily overlooked by a clinician. The importance of establishing a diagnosis here would lead to reduction in disease related mortality by prompt initiation of chemotherapy and supportive management.

\section{Case History}

A 79-year-old elderly diabetic male on regular medications presented to our outpatient department, being referred from an outside clinic for thrombocytopenia. He complained of a feeling of increased tiredness and generalised weakness for the past few months. He had no other complaints. He was accompanied by his two sons who also said that his physical activity had become very limited and he appeared to be lethargic. He had noticeable but unquantifiable weight loss and loss of appetite. He was on regular anti-diabetic drugs. He consumed mixed diet. Clinical examination revealed normal vitals. Examination of the respiratory system revealed 
reduced air entry in the left infra-scapular region. Other systems were unremarkable in terms of clinical examination.

Routine blood tests done showed anaemia ( $\mathrm{Hb}-$ $8 \mathrm{gm} / \mathrm{dl})$, thrombocytopenia (40,000), B12 deficiency $(92 \mathrm{pg} / \mathrm{ml})$ and a mildly elevated serum creatinine $(1.4 \mathrm{mg} / \mathrm{dl})$ with normal BUN. Peripheral smear done showed normocytic normochromic anaemia and thrombocytopenia. Initial Chest X-ray showed bilateral pleural effusion $(\mathrm{L}>\mathrm{R})$. A CT scan was done which revealed a lytic lesion with suspicious bone erosions in the L4 vertebral body, minimal loculated right pleural effusion with bronchiectatic changes of the right middle lobe, moderate left sided pleural effusion and diffuse osteopenia with degenerative changes in the spine. Electrolytes revealed hypercalcemia $(13 \mathrm{mg} / \mathrm{dl})$ with near normal Phosphate $(4.8 \mathrm{mg} / \mathrm{dl})$, low PTH $(2.7 \mathrm{pg} / \mathrm{ml})$, normal Vitamin $\mathrm{D}(18 \mathrm{ng} / \mathrm{ml})$ and normal Alkaline phosphate (50U/L) levels. A Lateral X-ray view of the LS Spine revealed diffuse osteosclerotic changes. Viral markers were non-reactive.

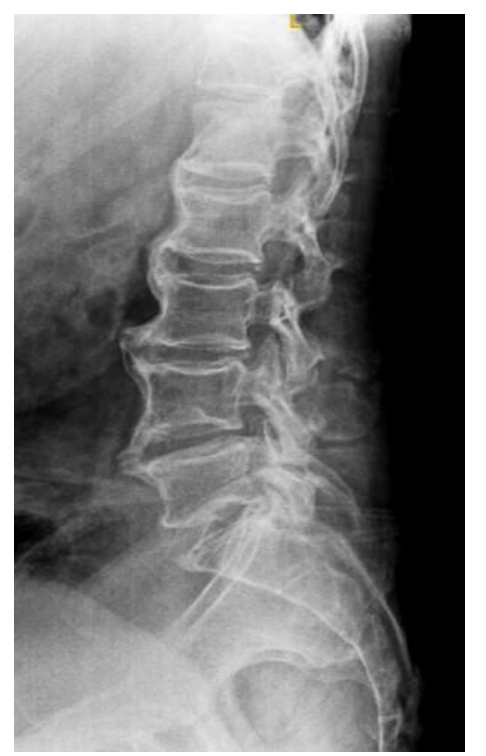

Figure 1 - Osteosclerotic changes of LS spine We proceeded to do a protein electrophoresis with the suspicion of plasma cell disorder in mind which showed a monoclonal band in the Beta 1 region $-55.8 \%$. A bone marrow biopsy done showed presence of sheets of plasma cells with scattered normal hematopoietic cells, IHC staining was done and turned out to be positive for CD138 and Lambda which concluded the diagnosis of multiple myeloma with lambda predominance.


Figure 2 - Bone marrow picture showing predominance of plasma cells

An X-Ray skull showed presence of a few punched out lytic lesions suggestive of myeloma.

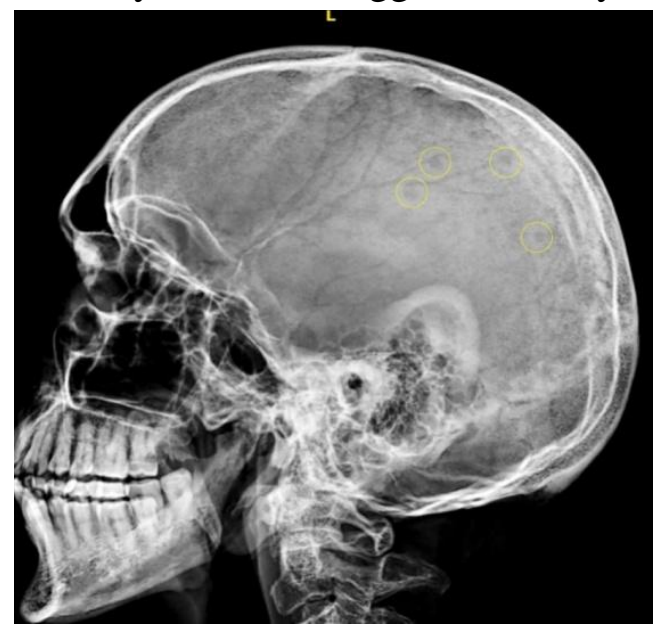

Figure 3 - X-Ray Skull showing multiple lytic lesions.

However, this did not explain the presence of pleural effusion. Could it be related to the plasma cell disorder? Or could it be a separate entity all together - ? A nerve conduction studies of all 4 limbs was done and showed severe sensory motor radiculopathy of all 4 limbs sparing bilateral median sensory nerves. The presence of a plasma cell disorder, polyneuropathy, mixed osteosclerotic, lytic lesions and pleural effusion, fulfilled the criteria for POEMS syndrome. Haematologist was consulted and patient was started on Thalidomide 100mg BD, Intravenous dexamethasone 40mg once weekly and Inj. Bortezomib. A repeat Chest X-ray after 2 weeks of therapy showed resolution of the Pleural effusion. 


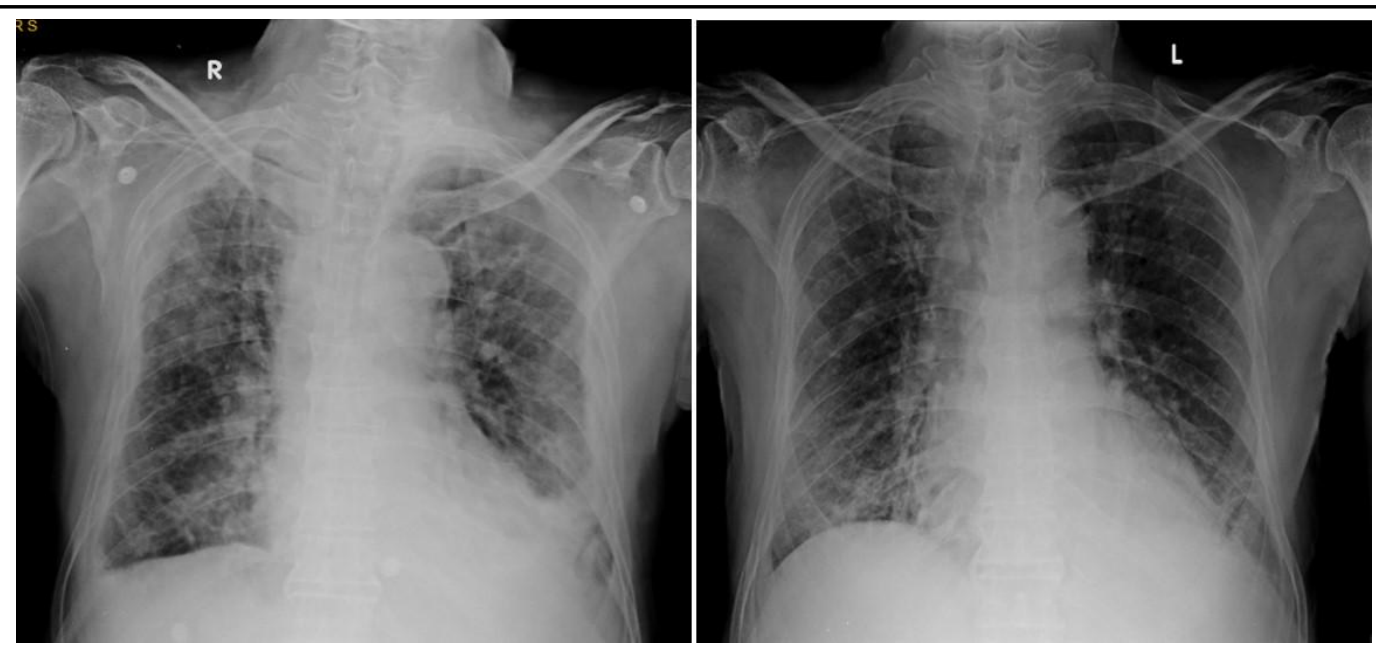

Figure 4 - Chest X-ray before and after initiation of treatment

During the course of hospital stay platelet count dropped further and hematuria manifested for which platelet transfusions were given. Patient still remains under our care for chemotherapy and after 3 weeks of treatment clinical improvement isn't very significant.

\section{Discussion}

POEMS Syndrome, also called as Crow-Fukase syndrome, osteosclerotic myeloma, PEP (Plasma cell dyscrasia, endocrinopathy and polyneuropathy) syndrome or Takatsuki syndrome, was first described in a patient back in the year 1938 who had sensorimotor peripheral neuropathy, hyperpigmentation, elevated cerebrospinal fluid protein, and a solitary plasmacytoma. Although the acronym POEMS was suggested for the presentation only later in 1980. The acronym expands itself as Polyneuropathy, Organomegaly, Endocrinopathy, Monoclonal protein and Skin changes. The disease commonly presents in the fifth to sixth decade of life with a male predominance ${ }^{1}$. Though the exact cause is still unknown it has been hypothesized that increased levels of proinflammatory cytokines such as interleukin-1ß (IL-1ß), tumour necrosis factor alpha (TNFalpha), and interleukin-6 (IL-6) play an important role in the pathogenesis ${ }^{2}$. The International Myeloma Working Group (IMWG) criteria has been formulated based on various case series and is currently used for the diagnosis of POEMS syndrome. It consists of a mandatory criteria, major criteria and minor criteria. Our patient had satisfied the criteria for diagnosis as he had a biopsy proven monoclonal plasma cell proliferative disorder and severe sensorimotor polyneuropathy under the mandatory criteria, a mixed osteolytic/sclerotic lesion under the major criteria and extravascular fluid overload manifestation (Pleural Effusion) for the minor criteria $^{3}$.

\begin{tabular}{|l|}
\hline Mandatory Criteria \\
\hline Polyneuropathy \\
\hline Monoclonal plasma cell proliferative disorder (almost always lambda) \\
\hline Major Criteria (One required) \\
\hline Sclerotic bone lesions \\
\hline Castleman's disease \\
\hline Elevated levels of vascular endothelial growth factor (VEGF) \\
\hline Minor Criteria (One required) \\
\hline Organomegaly (splenomegaly, hepatomegaly, or lymphadenopathy) \\
\hline Extravascular volume overload (edema, pleural effusion, or ascites) \\
\hline Endocrinopathy (adrenal, thyroid, pituitary, gonadal, parathyroid, pancreatic) \\
\hline Skin changes \\
\hline Papilledema \\
\hline Thrombocytosis/polycythemia \\
\hline
\end{tabular}

Table 1 - IMWG criteria for PEOMS syndrome Elevated VEGF levels in patients with POEMS syndrome is important as it is implicated in the pathogenesis of the syndrome itself ${ }^{4}$ but we hadn't estimated its level in our patient because the development of polyneuropathy in POEMS syndrome is thought to be VEGF related. The presence of monoclonal plasma cell disorder is almost always present in POEMS and predominantly lambda type of light chain as seen in our patient ${ }^{5}$. It is known that the M Band on 
protein electrophoresis is usually seen in the Gamma region but patient showed the $M$ band spike in the Beta-1 regions which is less common. According to a study conducted in Tunisia out of 242 patients with monoclonal gammopathy peak on the electrophoresis was seen in the gamma region in $78 \%$ of cases and in the beta region in $22 \%$ of cases ${ }^{6}$.

The incidence of pleural effusion in the setting of POEMS is about $28 \%^{7}$, which was present in our patient. In another follow-up study it was found that 5 of 20 patients with POEMS had developed pulmonary hypertension in a 10-year follow-up, our patient too at presentation had mild pulmonary artery hypertension $(42 \mathrm{mmHg})$, probably owing to the over expression of pro-inflammatory cytokines $^{8}$.

It is common for patients with POEMS to manifest with Thrombocytosis and Vitamin B12 deficiency but our patient had both anemia and thrombocytopenia but peripheral smear picture did not correlate that with which is seen in the setting of vitamin B12 deficiency. We suggest a probable immune mediated condition or could be due the bone suppression seen in a plasma cell dyscrasia.

Patients with a limited disease are usually managed with radiotherapy but ours was an advanced disease and hence we opted for chemotherapy. A study from china has shown a response rate of $76 \%$ with the use of bortezomib ${ }^{9}$ despite its neurotoxic effects hence we went with a combination of thalidomide, Dexamethasone and Bortezomib. Given the age of the patient the choice of hematopoietic cell transplantation is decided against.

\section{References}

1. Dispenzieri A et al. POEMS syndrome: definitions and long-term outcome. Blood 2003; 101:2496.

2. Gherardi RK et al. Overproduction of proinflammatory cytokines imbalanced by their antagonists in POEMS syndrome. Blood 1996; 87:1458.
3. Rajkumar SV et al. International Myeloma Working Group updated criteria for the diagnosis of multiple myeloma. Lancet Oncol 2014; 15:e538.

4. Watanabe $\mathrm{O}$ et al. Overproduction of vascular endothelial growth factor/vascular permeability factor is causative in CrowFukase (POEMS) syndrome. Muscle Nerve 1998; 21:1390.

5. Abe $\mathrm{D}$ et al. Restrictive usage of monoclonal immunoglobulin lambda light chain germline in POEMS syndrome. Blood 2008; 112:836.

6. Mseddi-Hdiji $\mathrm{S}$ et al. Monoclonal gammapa-thies in Tunisia: epidemiological, immunochemical and etiological analysis of 288 cases. Pathol Biol (Paris) 2005;53:19-25.

7. Allam JS et al. Pulmonary manifestations in patients with POEMS syndrome: a retrospective review of 137 patients. Chest 2008; 133:969.

8. Rose $\mathrm{C}$ et al. POEMS syndrome: report on six patients with unusual clinical signs, elevated levels of cytokines, macrophage involvement and chromosomal aberrations of bone marrow plasma cells. Leukemia 1997; 11:1318.

9. He H et al. Successful treatment of newly diagnosed POEMS syndrome with reduced-dose bortezomib based regimen. Br J Haematol 2018; 181:126. 